gegen die effektive Reninaktivität gemessen, für die neben der absoluten Reninaktivität die endngene Angiotensinogenkonzentration und Renin-Inhibitoren im Plasma bedeutsam sein können. Die Existenz von ReninInhibitoren im Plasma wird zwar immer wieder vermutet, konnte aber bisher nicht wahrscheinlich gemacht werden (13). Bei ausreichender endogener Angiotensinogenkonzentration und Fehlen von Inhibitoren ist die effektive gleich der absoluten Reninaktivität. Dementsprechend haben auch die Bestimmungen der absoluten (Methode Brown oder Gould) und der effektiven (Methode Boucher) Reninaktivität bei Gesunden und Kranken zu praktisch gleichen Ergebnissen geführt (ein direkter Vergleich der mit diesen $3 \mathrm{Me}-$ thoden erhaltenen Werte ist wegen der unterschiedlichen Definition der Enzym-Einheiten und Versuchsbedingungen nicht möglich). Unterschiedliche Werte für die effektive und die absolute Reninaktivität müssen dann erwartet werden, wenn zwar die absolute Reninaktivität hoch, die endogene Angiotensinogenkonzentration aber sehr niedrig ist. Eine solche Konstellation wurde bisher nur ganz selten bei Leberzirrhosen beobachtet (13), bei denen dann mit den Methoden von Brown oder von Gould eine hohe absolute, mit der Methode von Boucher eine niedrige effektive Reninaktivität ermittelt wird. Gerade für die Beurteilung auch dieser Fälle scheint uns aber die Methode von BOUCHER dem Verfahren von BROWN oder von Gould überlegen, weil hierbei eine niedrige effektive Plasmareninaktivität die Aktivität des Renin-AngiotensinSystems besser widerspiegelt als der Nachweis einer erhöhten absoluten Reninaktivität, die infolge der starken Verminderung des Angiotensinogens in vivo nicht wirksam ist.

\title{
Literatur
}

1. Brown, J. J., D. L. Davies, A. F. Lever und J. I. S. Robertson, Canad. Med. Ass. J. 90, 201 (1964). - 2. Helmer, O. M., Canad. Mled. Ass. J. 90, 221 (1964). - 3. Veyrat, R., J. de Champlain, R. Boucher und J. Genest, Canad. Med. Ass. J. 90, 215 (1964). 4. SkEGGS, L. T., K. E. Lentz, J. R. KAHN und N. P. Shumway, J. Exper. Med. 18, 283 (1958). - 5. Montague, D., B. Riniker, H. Brunner und F. Gross, Amer. J. Physiol. 210, 591 (1966). 6. Helarer, O. M. und W. E. Judson, Circulation 27, 1050 (1963). -7. Klaus, D., H. Kaffarnik und H. Pfeir, Klin. Wschr. 41, 376 (1963). - 8. Yoshinaga, K., M. Aida, M. Maebashi, T. Sato, K. Abe und J. Mrwa, Tohoku J. Exper. Med. 80, 32 (1963). 9. Warzinski, R., Y. DeMirjJiAN und S. Hoobler, Canad. Med. Ass. J. 90, 225 (1964). - 10. Fascrolo, J. C., E. DE Viro, J. C. Romero und J. N. Cucchi, Canad. Med. Ass. J. 90, 206 (1964). 11. Lever, A. F., J. I. S. Robertson und M. Tree, Biochem. J. 91, 346 (1964). - 12. Brown, J. J., D. L. Davies, A. F. Lever, J. I. S. Roberrson und M. TREe, Biochem. J. 93, 594 (1964). -
13. Gould, A. B., L. T. SkEGGs und J. R. KAHN, Laborat. Invest. 15, 1802 (1966). - 14. Pickens, P. T., F. M. Bumpus, A. M. Lloyd, R. B. SMEBY und I. H. PAGE, Circulat. Res. 17, 438 (1965). - 15. Boucher, R., R. Vexrat, J. de Champlain und J. Genest, Canad. Med. Ass. J. 90, 194 (1964). - 16. Boucher, R. und J. Genest, Canad. J. Physiol. Pharmacol. 44, 181 (1966). - 17. Gross, F. und P. Lichtlen, Naunyn-Schmiedebergs Arch. exp. Pathol. Pharmakol. 233, 323 (1958). - 18. HaAs, E., H. Lamfrom und H. GoldBLATT, Arch. Biochem. Biophysics 48, 256 (1954). - 19. MAEbashi, M., K. Yoshinaga, M. Arpa, M. Okuyama und A. Orkawa, Tohoku J. Exper. Med. 85, 299 (1965). - 20. Helmer, O. M., Progr. Cardiovasc. Dis. 8, 117 (1965). - 21. Haynes, F. W. und L. Dexter, Federat. Proc. 2, 20 (1943). - 22. Klaus, D. und A. Herzmann, Dtsch. med. Wschr.. 46, 2114 (1967). - 23. Plentr, A. A. und I. H. PAge, J. Exper. Med. 78, 367 (1943). 24. Daum, A., D. Klaus und H. UehreEke, Naunyn-Schmiedebergs Arch. exp. Pathol. Pharmakol. 254, 327 (1966).

\section{Urinvolumen und Ausscheidung von Kreatinin, 17-Ketosteroiden und 17-Hydroxycorticosteroiden während experimenteller Änderung der Flüssigkeitsaufnahme}

\author{
Von R. Borth, H. Breuer und D. GütgemanN
}

Aus dem Laboratoire d'bormonologie, Clinique universitaire de gynécologie et d'obstétrique, Genève, Schweiz (Direktor: Prof. Dr. H. de Watteville) und der Abteilung für Klinische Chemie und Biocbemie der Cbirurgischen Universitätsklinik, BonnVenusberg (Direktor: Prof. Dr. A. Gütgemann)

(Eingegangen am 2. Mai 1967)

Bei vier gesunden männlichen Versuchspersonen im Alter von 23 bis 34 Jahren wurden das Urinvolumen, das spezifische Gewicht sowie die Ausscheidung der 17-Ketosteroide, der 17-Hydroxycorticosteroide und des Kreatinins im Urin während steigender Flüssigkeitszufuhr gemessen. Die Ergebnisse wurden mit Hilfe der Korrelations- und Regressionsrechnung statistisch ausgewertet. Die Konzentrationen waren den Volumina der 24-Stdn.-Urine umgekehrt proportional; die Regressionskoeffizienten lagen in der gleichen Größenordnung wie die bei einem reinen Verdünnungseffekt theoretisch zu erwartenden Werte. Eine Zunahme der 24-Stdn.-Mengen mit steigendem Urinvolumen ließ sich statistisch sichern, war aber zu geringfügig, um von praktischer Bedeutung zu sein.

Eine zuverlässige Kontrolle der Vollständigkeit der Sammlung eines' 24-Stdñ.-Urins ist weder durch Bestimmung des Kreatinins noch durch Messung des spezifischen Gewichts möglich. Verläßliche Steroidbestimmungen setzen vollständige Sammlung des 24-Stdn.-Urins voraus. 
The twenty-four-hour urine volume, the urine density and the urinary 17 -ketosteroids, 17 -hydroxycorticosteroids and creatinine were measured for four healthy males in the.age range 23-34 years, over a period in which the uptake of fluid was continually increased. Correlation and regression analysis were used in the statistical evaluation of the data. The concentrations decreased with increasing 24-hour urine volume; the regression coefficients were similar to those expected for a simple dilution model. Steroid excretion rates increased slightly with fluid volume; however, this effect - although not attributable to chance variation - was too small to be of practical significance.

Neither the determination of creatinine nor the measurement of density provide a reliable test for the completeness of 24-hour urine collection. The latter remains an essential requirement for accurate estimation of excretion rates of steroids.

Die Ausscheidung von 17-Ketosteroiden ${ }^{1}$ ) und 17Hydroxycorticosteroiden wird im allgemeinen in Milligrammen pro 24 Stdn. (mg/24 Stdn.) angegeben. Dabei bleibt das Urinvolumen unberücksichtigt in der Meinung, die Tagesausscheidung sei unabhängig von Flüssigkeitsaufnahme und -ausscheidung im Urin. Diese Annahme wird durch die Untersuchungen von KASSENAAR und Mitarbeitern (1), Hoyt und Levine (2), Lugg und Bowness (3) sowie Hamburger (4) gestützt. Demgegenüber soll nach anderen Autoren die Tagesausscheidung mit dem Urinvolumen zunehmen. Nach Devis (5) ist die 17-KS-Konzentration vom Urinvolumen unabhängig. HollANDER und Mitarbeiter (6) fanden eine hohe positive Korrelation $\left(r^{2}=0,52\right.$ bis 0,98$)$ zwischen Tagesvolumen und biologisch bestimmter Androgenausscheidung. MCHENRY und Mitarbeiter (7) $\left(r^{2}=0,24\right.$ bis 0,59$)$ sowie LAROCHE und Mitarbeiter (8) machten ähnliche Beobachtungen für die 17-KS. Falls eine solche Abhängigkeit wirklich besteht sowie im Hinblick auf die Fragwürdigkeit der vollständigen Sammlung von 24-Stdn.-Urinen ergibt sich die Frage, ob für die klinische Auswertung ein anderer Parameter nicht geeigneter ist. So ist einmal die Steroidkonzentration in mg pro Liter, zum anderen die pro Gramm Kreatinin berechnete Steroidmenge empfohlen und benutzt worden.

Als Beitrag zur Klärung dieser Fragen haben wir unter kontrollierten Versuchsbedingungen die Ausscheidung der 17-KS, der 17-OHCS und des Kreatinins in Abhängigkeit von der Flüssigkeitsbilanz bei gesunden männlichen Versuchspersonen studiert und die Beobachtungen statistisch ausgewertet. Dabei wurden folgende Fragestellungen geprüft:

1. Beeinflußt das Getränkevolumen die ausgeschiedene Tagesmenge?

2. Variiert die Tagesausscheidung des Kreatinins weniger als die der Steroidfraktionen?

3. Variiert die pro Gramm Kreatinin berechnete Steroidausscheidung weniger als die Tagesmenge?

4. Ist die Steroidkonzentration bei unbekanntem 24 Stdn.-Urinvolumen und unbekannter Sammelperiode ein Anhaltspunkt zur Bestimmung der Tagesmenge?

\section{Methodik}

Versuchspersonen und Versuchsanordnung

Viẹt gesunde berufstätige Männer im Alter von 23, 27, 31 bzw. 34 Jahren sammelten während der Monate Mai und Juni im Verlauf von $19,13,19$ bzw. 17 aufeinanderfolgenden Tagen sorgfältig den 24-Stdn.-Urin. Die Wasseraufnahme (Getränke) wurde in den

1) Abkulrungen: 17-KS $=$ 17-Ketosteroide; $17-$ OHCS $=17-$ Hydroxycorticosteroide. ersten Tagen stark eingeschränkt und dann progressiv gesteigert; die 24-Stdn.-Volumina stiegen während der Versuchsdauer von 0,6 auf 3,3 , von 0,9 auf 3,5 , von 0,5 auf $7,3 \mathrm{bzw}$. von 0,5 auf $2,8 l$. Alle Versuchspersonen schienen frei von endokrinen Störungen. Keiner der durchgeführten Leberfunktionsteste (Bilirubin, Elektrophorese, Eiweißlabilitätsproben, Serum-Glutamat-Pyruvat-Transaminase $^{1}$ ) und Cholinesterase) ergab pathologische Werte.

Urine

Am Ende jeder 24stdg. Sammelperiode wurden das Volumen (l/24 Stdn.) und das spezifische Gewicht bestimmt. Dann wurden die Urine in Kunststoff-Flaschen eingefroren und bis zur Aufarbeitung bei $-10^{\circ}$ aufbewahrt.

Bestimmung der 17-Ketosteroide

Die 17-KS wurden in $8 \mathrm{~m} l$ Urin nach NoryMrberski und Mitarbeitern (9) bestimmt, wobei jedoch auf den Zusatz von Essigsäure vor der Hydrolyse verzichtet wurde. Die ZimmermannReaktion wurde nach der Vorschrift von CALLOw und Mitarbeitern (10) in absolut-alkoholischer $\mathrm{KOH}$ unter Verwendung der Korrekturformel von TALBOT und Mitarbeitern (11) ausgeführt. Die Resultate wurden ausgedrückt in DehydroepiandrosteronÄquivalenten.

\section{Bestimmung der 17-Hydroxycorticosteroide}

Die totalen 17-OHCS wurden nach APpLEBY und Mitarbeitern (12) in $4 \mathrm{ml}$ Urin bestimmt; dabei wird der Urin mit Natriumborhydrid (Merck, Darmstadt) reduziert und anschließend mit Natriumwismutat (British Drug Houses) oxydiert. Die Resultate wurden ausgedrückt in Dehydroepiandrosteron-Äquivalenten.

\section{Bestimmung des Kreatinins}

Die Kreatininbestimmung erfolgte nach der Methode von Popper und Mitarbeitern (13). Zur Analyse wurde der Urin im Verhältnis 1: 50 mit Aqua bidest. verdünnt; dem verdünnten Urin wurden Pikrinsäure und Natronlauge zugesetzt, die Ablesung der Extinktion geschah nach $12 \mathrm{Min}$. bei $546 \mathrm{~m} \mu$ gegen einen Reagenzienleerwert. Die Resultate wurden in Kreatinin-Äquivalenten ausgedrückt.

\section{Statistische Methoden}

Regressions- und Korrelationsrechnungen sowie Streuungszerlegung wurden nach den von LINDER (14) beschriebenen Standardmethoden durchgeführt.

\section{Ergebnisse und statistische Auswertung}

Die bei den vier Versuchspersonen gemessenen Werte für das Urinvolumen, das spezifische Gewicht sowie die Ausscheidung der 17-KS, 17-OHCS und des Kreatinins sind in Tabelle 1 zusammengestellt. Bei der Betrachtung der Werte gewinnt man den Eindruck, daß bei den einzelnen Versuchspersonen die Ausscheidungswerte keine eindeutige Abhängigkeit vom Urinvolumen zeigen; das bedeutet eine Abnahme der spezifischen Gewichte und der Konzentrationen bei Zunahme des Volumens. Um diese Eindrücke $\mathrm{zu}$ objektivieren und weitere $\mathrm{Zu}$ sammenhänge aufzudecken, wurde die folgende statisti-

1) Der Trivialname Glutamat-Pyruvat-Transaminase wird hier gebraucht für das Enzym L-Alanin : 2-oxoglutamat Aminotransferase EC 2.6.1.2, Cholinesterase für Acetylcholin Acylhydrolase EC 3.1.1.8. 


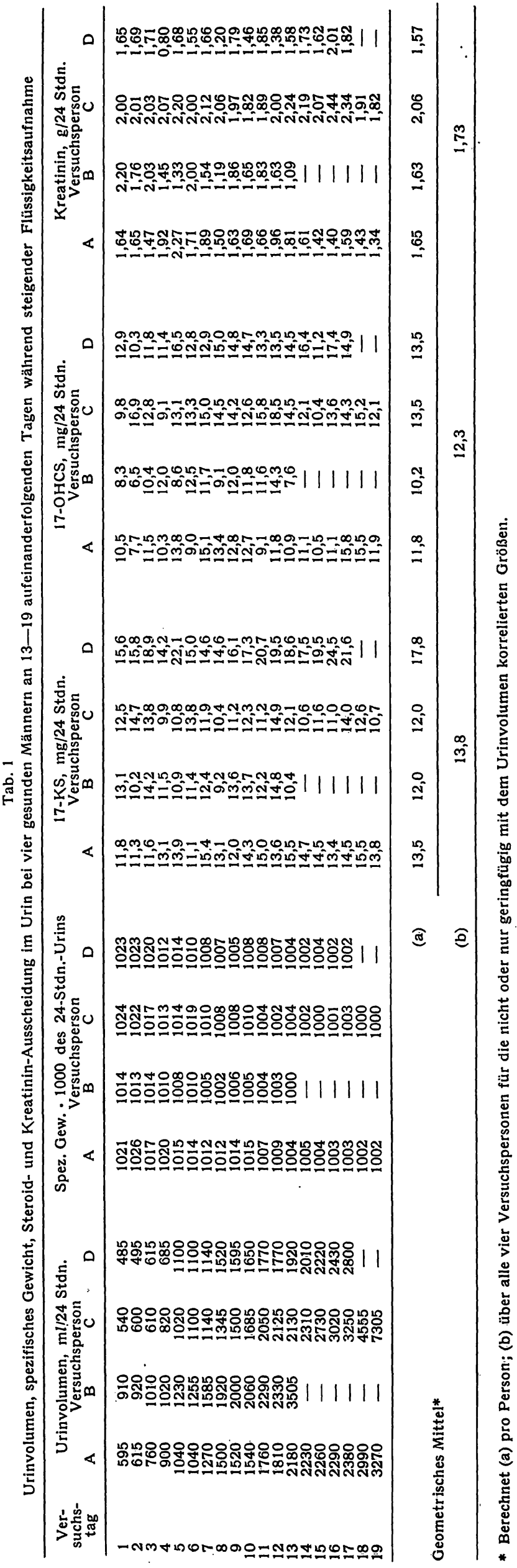

sche Analyse durchgeführt. Die dabei benutzten Methoden seien durch einige Vorbemerkungen erläutert.

Viele Beobachtungen sprechen dafür, daß bei biologischen Größen relative Änderungen maßgebender sind als absolute Differenzen, und daß solche Größen oft lognormal verteilt sind. Werden die hier gemessenen Werte gegen die Urinvolumina im doppelt-logarithmischen Maßstab aufgetragen, so scheinen die Punkte um gerade Linien zu streuen; für das spezifische Gewicht trifft dies bereits angenähert $\mathrm{zu}$, wenn es ohne logarithmische Transformierung gegen die Logarithmen der Urinvolumina aufgetragen wird.

Aus den genannten Gründen wurden alle statistischen Rechnungen nach linearer Transformierung des spezifischen Gewichts und logarithmischer Transformierung aller anderen Meßwerte durchgeführt. Für diese Meßwerte wurden folgende Einheiten verwendet:

1. Urinvolumen in $\mathrm{m} l / 24 \mathrm{Stdn}$.

2. spezifisches Gewicht

3. Kreatinin-Konzentration in $\mathrm{g} / \mathrm{l}$

4. Kreatinin-Ausscheidung in $\mathrm{g} / 24 \mathrm{Stdn}$.

5. 17-KS-Konzentration in $\mathrm{mg} / \mathrm{l}$

6. 17-KS-Ausscheidung in $\mathrm{mg} / 24 \mathrm{Stdn}$.

7. 17-KS-Ausscheidung in $\mathrm{mg} / \mathrm{g}$ Kreatinin

8. 17-OHCS-Konzentration in $\mathrm{mg} / \mathrm{l}$

9. 17-OHCS-Ausscheidung in $\mathrm{mg} / 24 \mathrm{Stdn}$.

10. 17-OHCS-Ausscheidung in $\mathrm{mg} / \mathrm{g}$ Kreatinin.

Ist $w$ ein Meßwert in den angegebenen Einheiten und $x$ (Urinvolumen) bzw. $y$ (alle anderen Angaben) der entsprechende transformierte Rechenwert, so gilt

1. für das Urinvolumen $x=100 \log (w / 100)$

2. für das spez. Gewicht $y=1000(y-1)$

3. für die Kreatininkonzentration $y=100 \log (10 w)$

4. bis 10. für alle anderen Werte $y=100 \log w$

Auf diese Weise erhält man ein- bis dreistellige Zahlen, mit denen gut zu rechnen ist. Der einheitlichen Interpretation wegen wurden diese Transformationen auch dort verwendet, wo ihr Einfluß auf die Auswertung vermutlich nur sehr geting ist. Es sei erwähnt, daß beim Rechnen mit logarithmisch transformierten Größen aus algebraischen Summen - z. B. $\overline{\mathrm{x}} \pm \mathrm{s}$ (arithmetisches Mittel und Standardabweichung) - Produkte und Quotienten - z. B. $m \cdot p^{ \pm 1}$ - werden.

Das Urinvolumen wurde als unabhängige, experimentell gesetzte Variable angesehen. Für jede der übrigen neun Wertegruppen wurden die linearen Regressionskoeffizienten $(b)$ und die Quadrate der Korrelationskoeffizienten $\left(r^{2}\right.$; sog. Bestimmtheitsmaße, vgl. (14)) berechnet. Durch Streuungszerlegung wurde geprüft, ob die berechneten Abhängigkeiten mit einiger Wahrscheinlichkeit als real, d. h. nicht zufällig, gelten können und ob die Regressionsgeraden der vier Versuchspersonen angenähert parallel sind. Durchschnittswerte von $b$ und $r^{2}$ innerhalb aller vier Versuchspersonen wurden ebenfalls berechnet; sie sind jedoch, streng genommen, nur für die homogenen Daten sinnvoll. Für die nicht oder nur geringfügig mit dem Urinvolumen korrelierten Größen wurde ein wahrscheinlicher Streubereich $(P=0,9)$ berechnet. Schließlich wurden die Streubereiche ausgedrückt in Prozent der geometrischen Mittelwerte und die Regressionskoeffizienten als absolute (spezifisches Gewicht) bzw. prozientuale (alle übrigen Gruppen) Änderung des Regressionswertes bei Zunahme des Urinvolumens um $10 \%$. 
Die Ergebnisse der statistischen Auswertung sind in den Tabellen 2 und 3 und in den Abbildungen 1-6 dargestellt. Wie aus Tabelle 2 und Abbildung 1 hervorgeht, nimmt bei allen vier Versuchspersonen das spezifische Gewicht mit steigendem Urinvolumen ab, und zwar um

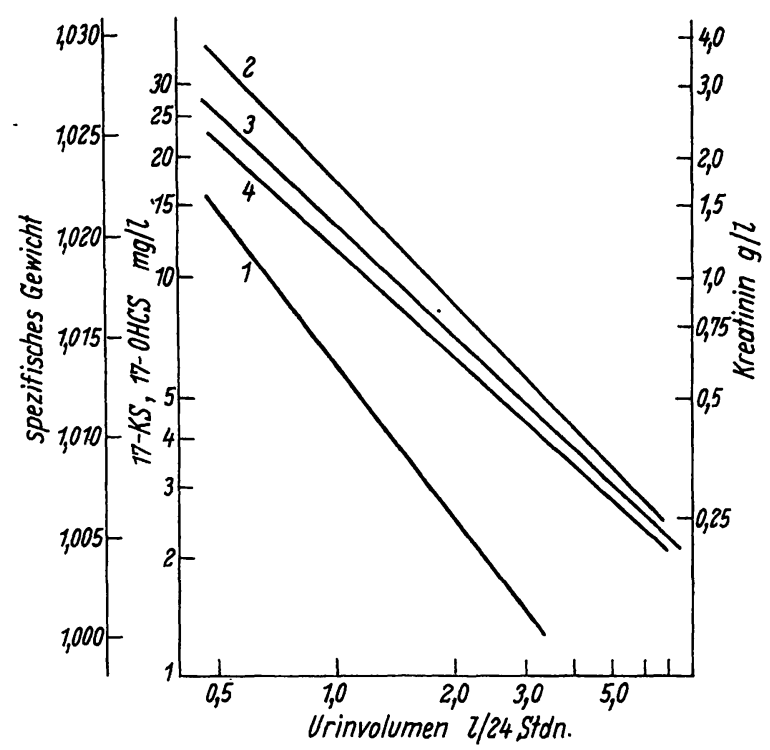

Abb. 1

Durchschnittliche Regressionsgeraden für die Abhängigkeit des spezifischen Gewichts (Kurve 1) sowie der Konzentration des Kreatinins (Kurve 2), der 17-Ketosteroide (Kurve 3) und der 17-Hydroxycorticosteroide (Kurve 4) vom 24-Stdn.-Volumen des Urins. Die Steigung der Geraden entspricht den mittleren Regressionskoeffizienten der Tabelle 2. eine Einheit in der dritten Dezimalstelle bei Vergrößerung der Flüssigkeitsmenge um 10\%. Die Korrelationen sind hoch und können $83-91 \%$ der Schwankungen des spezifischen Gewichtes (vgl. Tab. 2) erklären. Auch die Konzentrationen der 17-KS, der 17-OHCS und des Kreatinins nehmen mit steigendem Urinvolumen ab, und zwar um $8-10 \%$ bei Ansteigen des Tagesvolumens um $10 \%$. Die Korrelationen sind ebenfalls hoch und können 72-99\% der Konzentrationsschwankungen (vgl. Tab. 2) erklären.

Die ausgeschiedenen Tagesmengen sind vom Urinvolumen nahezu, jedoch nicht völlig, unabhängig, und es bestehen anscheinend einige Unterschiede sowohl $z$ wischen den Versuchspersonen als auch zwischen den Fraktionen.

So steigt bei zwei Versuchspersonen die Ausscheidung der 17-KS um etwa $1,5 \%$ an, wenn die Urinmenge um $10 \%$ zunimmt; diese Korrelationen können in einem Fall 52, im anderen 29\% der Tagesschwankungen erklären. Im Durchschnitt aller vier Fälle beträgt diese Zunahme 0,6\%; die Gesamtkorrelation erklärt $6 \%$ der Schwankungen (Tab. 2 und Abb. 2). Die Ausscheidung der 17OHCS steigt bei einer Versuchsperson um 1,6\% an, wenn die Urinmenge um 10\% zunimmt, und dieser Zusammenhang kann 37\% der Tagesschwankungen erklären. Obwohl die Streuung bei den drei anderen Fällen zu groß ist, um einen Zusammenhang zu sichern, ergibt sich im Durchschnitt der vier Fälle eine Zunahme der $17-\mathrm{OHCS}$ um $1 \%$ bei Volumenzunahme um $10 \%$; diese Korrelation kann für $10 \%$ der gesamten Tagesschwankungen verantwortlich gemacht werden (Tab. 2 und Abb. 3). Bei keiner Versuchsperson ergibt sich ein Anhaltspunkt für einen Zusammenhang zwischen Kreatininausscheidung und Urinmenge (Tab. 2 und Abb. 4).

Tab. 2

Regressions- und Korrelationskoeffizienten für die Abhängigkeit des spezifischen Gewichts, des Kreatinins, der 17-Ketosteroide und der 17Hydroxycorticosteroide vom Urinvolumen

$b \%=$ Prozentuale Änderung des Regressionswertes bei Zunahme des Urinvolumens um $10 \%+$. Nur beim spezifischen Gewicht handelt es sich

$r^{2}=$ um absolute (nicht prozentuale) Anderungen. $* *$ zwischen 0,01 und $0,001, * * *$ unter 0,001 . Für die übrigen Werte ist $P>0,05$, sie sind (nach diesern Kriterium) nicht von Null unterscheidbar. Diese Ergebnisse der statistischen Prüfung gelten auch für die entsprechenden Regressionskoeffizienten.

\begin{tabular}{|c|c|c|c|c|c|c|c|c|c|c|}
\hline \multirow[b]{2}{*}{ Meßwert } & \multirow{2}{*}{\multicolumn{2}{|c|}{ A }} & \multicolumn{2}{|c|}{ B } & \multicolumn{2}{|c|}{ Versuchspersonen } & \multicolumn{2}{|c|}{ D } & \multicolumn{2}{|c|}{ Alle vier } \\
\hline & & & $b \%$ & $r^{2}$ & $b \%$ & $r^{2}$ & $b \%$ & $r^{2}$ & $b \%{ }^{\text {Alle }}$ & ier $r^{2}$ \\
\hline Spezifisches Gewicht & $-0,0013^{x}$ & $0,91 * * *$ & $-0,0010^{x}$ & $0,89 * * *$ & $-0,0010 x$ & $0,83^{* * *}$ & $-0,0011^{x}$ & $0,90 * * *$ & $-0,0011 x$ & $0,86 * * *$ \\
\hline 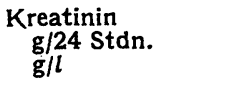 & $\begin{array}{l}-1,0 \\
-1,0,0\end{array}$ & $\begin{array}{l}0,15 \\
0,95^{* * *}\end{array}$ & $\begin{array}{l}-2,4 \\
-11,3\end{array}$ & $\begin{array}{l}0,26 \\
0,90 * * *\end{array}$ & $\begin{array}{c}0 \\
-9,1\end{array}$ & $\begin{array}{l}0 \\
0,99 * * *\end{array}$ & $\begin{array}{l}+1,0 \\
-8,1\end{array}$ & $\begin{array}{l}0,08 \\
0,86 * * *\end{array}$ & $\begin{array}{l}-0,23^{+} \\
-9,3^{+}\end{array}$ & $\begin{array}{l}0,01 \\
0,93 * * *\end{array}$ \\
\hline $\begin{array}{l}\text { 17-KS } \\
\mathrm{mg} / 24 \mathrm{Stdn} . \\
\mathrm{mg} / \mathrm{l} \\
\mathrm{mg} / \mathrm{g} \text { Kreatinin }\end{array}$ & $\begin{array}{l}+1,5 \\
-7,7 \\
+2,4\end{array}$ & $\begin{array}{l}0,52 * * * \\
0,97 * * * \\
0,60 * * *\end{array}$ & $\begin{array}{c}0 \\
-9,1 \\
+2,4\end{array}$ & $\begin{array}{l}0 \\
0,89 * * * \\
0,43 *\end{array}$ & $\begin{array}{l}-0,4 \\
-9,3 \\
-0,3\end{array}$ & $\begin{array}{l}0,04 \\
0,97 * * * \\
0,02\end{array}$ & $\begin{array}{l}+1,6 \\
-7,7 \\
+0,5\end{array}$ & $\begin{array}{l}0,29 * \\
0,92 * * * \\
0,03\end{array}$ & $\begin{array}{l}+0,6^{+} \\
8,6^{+} \\
+0,8^{+}\end{array}$ & $\begin{array}{l}0,06 * \\
0,94 * * * \\
0,08 *\end{array}$ \\
\hline $\begin{array}{l}\text { 17-OHCS } \\
\mathrm{mg} / 24 \text { Stdn. } \\
\mathrm{mg} / \mathrm{l} \\
\mathrm{mg} / \mathrm{g} \text { Kreatinin }\end{array}$ & $\begin{array}{l}+1,6 \\
-7,7 \\
+2,6\end{array}$ & $\begin{array}{l}0,19 \\
0,86 * * * \\
0,35 * *\end{array}$ & $\begin{array}{r}+1,2 \\
+7,9 \\
+3,7\end{array}$ & $\begin{array}{l}0,06 \\
0,72 * * * \\
0,37 *\end{array}$ & $\begin{array}{l}+0,4 \\
+8,7 \\
+0,5\end{array}$ & $\begin{array}{l}0,03 \\
0,93 * * * \\
0,03\end{array}$ & $\begin{array}{l}+1,6 \\
+7,7 \\
+0,3\end{array}$ & $\begin{array}{l}0,37 * * \\
0,94 * * * \\
0,01\end{array}$ & $\begin{array}{l}+1,0^{+} \\
\begin{array}{l}8,1^{+} \\
+1,2^{+}\end{array}\end{array}$ & $\begin{array}{l}0,10 * * \\
0,89 * * * \\
0,11 * *\end{array}$ \\
\hline
\end{tabular}

+ Diese Durchschnittswerte sind, streng genommen, statistisch nicht sinnvoll, da die Differenzen zwischen den vier Einzelwerten zu groß sind Diese Problemstellung nicht ins Gewicht fallen.

Tab. 3

Wahrscheinliche Streubereiche $(P=0,9)$ des Kreatinins, der 17-Ketosteroide und der 17-Hydroxycorticosteroide nach Vernachlässigung der Abhängigkeit vom Urinvolumen. Die Angaben erfolgen in Prozent der entsprechenden geometrischen Mittelwerte

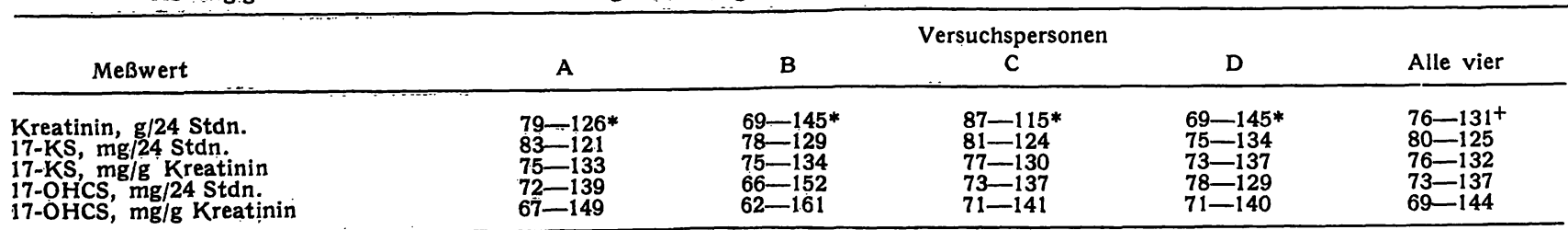

* Die Streubereiche der vier Personen sind gesichert voneinander verschieden. Siehe auch die Anmerkung + zu Tabelle 2. 


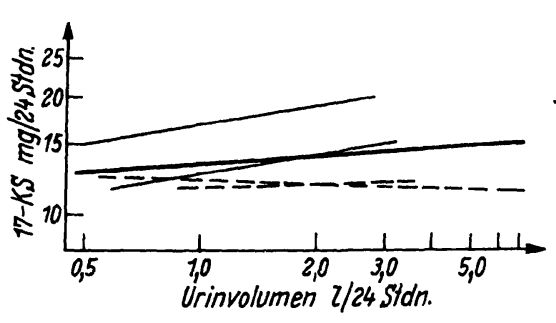

Abb. 2

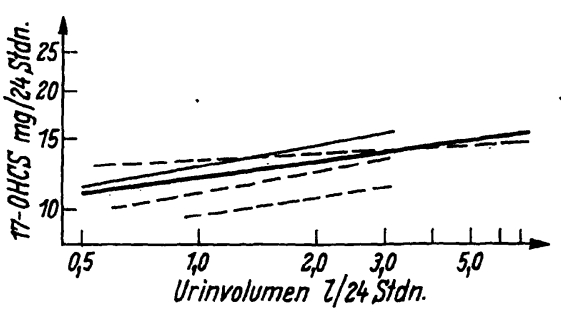

Abb. 3

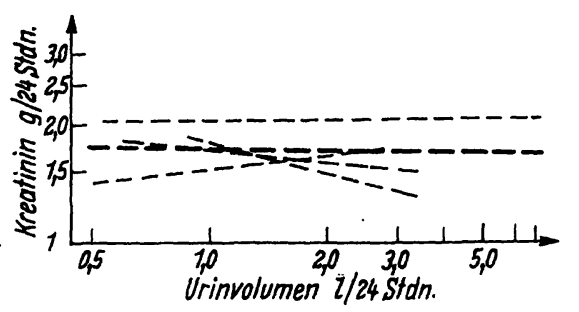

Abb. 4

Abb. 2. Regressionsgeraden für die Abhängigkeit der 17-Ketosteroid-Ausscheidung vom 24.-Stdn.-Volumen des Urins. Die vier dünnen Geraden entsprechen den vier Versuchspersonen; ist die Gerade gestrichelt, so war die Korrelation unsicher oder nicht vorhanden $(P>0,05 ;$ vgl. Tab. 2). Die fette Gerade entspricht der durchschnittlichen Regression innerhalb der vier Versuchspersonen. Die Länge der Geraden zeigt den Beobachtungsbereich an.

Abb. 3. Regressionsgeraden für die Abhängigkeit der 17-Hydroxycorticosteroid-Ausscheidung vom 24-Stdn.-Volumen des Urins. Darstellung wie in Abbildung 2.

Abb. 4. Regressionsgeraden für die Abhängigkeit der Kreatinin-Ausscheidung vom 24-Stdn.-Volumen des Urins. Darstellung wie in Abbildung 2.

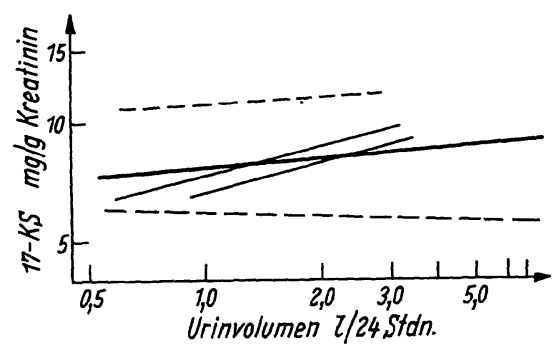

Abb. 5

Regressionsgeraden für die Abhängigkeit der pro Gramm Kreatinin ausgedrückten 17-Ketosteroid-Ausscheidung vom 24-Stdn.Volumen des Urins. Darstellung wie in Abbildung 2.

Wird die Steroidausscheidung pro Gramm Kreatinin berechnet, so zeigen diese Quotienten ein ähnliches Verhalten wie die Tagesmengen; das Ansteigen mit der Urinmenge ist ab er etwas deutlicher ausgeprägt.

In zwei Fällen nimmt die so ausgedrückte Ausscheidung der 17-KS um 2,4\%, im Durchschnitt aller Fälle um $0,8 \%$ zu bei Volumenzunahme um $10 \%$. Die entsprechenden Bestimmtheitsmaße sind $0,60,0,43$ und 0,08 , und der Zusammenhang kann also diese Bruchteile der Gesamtstreuung erklären (Tab. 2 und Abb. 5). Die Ausscheidung der 17-OHCS steigt in zwei Fällen um 2,6 bzw. 3,7\%, im Durchschnitt um 1,2\% an bei Zunahme des Urinvolumens um $10 \%$; diese Zusammenhänge können 35, 37 und 11\% der Streuung erklären (Tab. 2 und Abb. 6).

Sieht man von den oben beschriebenen schwachen Korrelationen mit dem Urinvolumen $a b$, so führt eine Betrachtung der Streubereiche der Tagesschwankungen zu folgenden Ergebnissen (Tab. 3).

1. Die Tagesausscheidung des Kreatinins schwankt im Durchschnitt in derselben Größenordnung wie die der Steroidfraktionen; der relative Streubereich ist aber individuell verschieden.

2. Die pro 24 Stdn. berechneten Steroidmengen variieren eher weniger als die pro Gramm Kreatinin berechneten Werte.

\section{Diskussion und Schlußfolgerungen}

Der hier beobachtete enge Zusammenhang zwischen Urinvolumen und Konzentration entspricht einem reinen Verdünnungseffekt. Bei einem solchen sind die beiden Größen einander umgekehrt proportional; einer $\mathrm{Zu}$ -

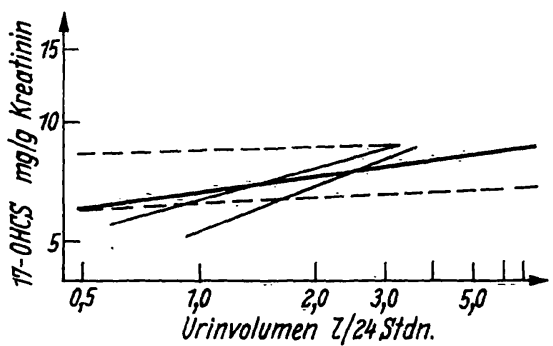

Abb. 6

Regressionsgeraden für die Abhängigkeit der pro Gramm Kreatinin ausgedrückten 17-Hydroxycorticosteroid-Ausscheidung vom 24-Stdn.-Volumen des Urins. Darstellung wie in Abbildung 2.

nahme des Urinvolumens um $10 \%$ entspricht daher theoretisch eine Abnahme der Konzentration um 9,1\%. Unsere Werte streuen für die verschiedenen Personen und Fraktionen zwischen 7,7 und $11,3 \%$ und bewegen sich damit in der Nähe des theoretischen Wertes. Dieses Ergebnis spricht erstens dafür, da $\beta$ die glomeruläre Filtration und die tubuläre Rückresorption unter unseren Versuchsbedingungen keine Abweichungen von der Norm zeigten. Zweitens läßt es erkennen, daß die Steroidkonzentration - im Unterschied zur Ausscheidungsrate - als endokrinologischer Parameter nicht brauchbar ist.

Derselbe Verdünnungseffekt zeigt sich bei den spezifischen Gewichten ebenso ausgeprägt wie bei den Konzentrationen; dies ist nicht erstaunlich, spiegelt doch das spezifische Gewicht die Gesamtheit aller im Urin gelösten Stoffe wider. Die Suche nach einem Test, mit dessen Hilfe die Vollständigkeit einer 24-Stdn.-Urinsammlung geprüft werden kann, läuft auf folgende Frage hinaus: Wird im Urin ein Stoff in genügend konstanter Tagesmenge ausgesschieden, so $\mathrm{da} \beta$ von seiner Konzentration auf das zu erwartende 24-Stdn.-Volumen geschlossen werden kann? Kreatinin ist zu diesem Z weck empfohlen worden. Man kann auch daran denken, das spezifische Gewicht in diesem Sinne zu verwenden, wie dies von Medgyesr und Katona (15) vorgeschlagen worden ist. Nach unseren Beobachtungen ist der Aussagewert beider Parameter gleichermaßen begrenzt, da nach der Streuungsbreite der als ,hinreichend konstant" vorauszusetzenden Größen - Tágesmenge des Krea- 
tinins (Tab. 3) bzw. aller gelösten Urinbestandteile oder eine entsprechende aus der Regressionsgleichung abgeleitete Größe - der Unsicherheitsfaktor zu groß ist. Man darf darüber hinaus nicht übersehen, daß die $\mathrm{Ab}$ hängigkeit der Ausscheidung von der Tageszeit, über die unsere Beobachtungen nichts aussagen, bei diesem Problem eine große Rolle spielt.

Daher hat es auch wenig Sinn, die Steroidausscheidung pro Gramm Kreatinin auszudrücken. Wie Tabelle 3 zeigt, sind die Schwankungsbreiten dieses Parameters durchaus nicht kleiner als die der 24-Stdn.-Mengen. Aus dem Gesagten geht hervor, daß die möglichst genaue Bestimmung der Sammelperiode sowie verlustloses Sammeln des Urins weiterhin als unerläßliche Voraussetzungen zum Erzielen sinnvoller Analysenresultate anzusehen sind.
Die geringfügige, wenn auch statistisch gesicherte $\mathrm{Zu}$ nahme der Steroidausscheidung mit dem Urinvolumen konnte nur auf Grund der extremen Versucbsbedingungen erkannt werden. Diese Zunahme dürfte jedoch im normalen Schwankungsbereich der Flüssigkeitsmenge zu vernachlässigen sein. Aus diesen Gründen kommt dem Phänomen, trotz seiner statistischen Sicherung, keine praktische Bedeutung zu. Hier zeigt sich erneut, daß bei der Interpretation biologischer Erscheinungen die statistische Signifikanz sehr wohl von der Relevanz des beobachteten Effektes unterschieden werden muß. Diese Feststellung schließt jedoch keineswegs die Frage aus, ob der hier beobachtete Zusammenhang auf einem Stoffwechselphänomen oder auf einer erhöhten Sekretion beruht. Bei solchen Überlegungen wird die Rolle der Niere zu berücksichtigen sein.

\title{
Literatur
}

1. Kassenaar, A., J. Huis in't Veld, P. Siderius, H. C. Seldenrath und A. Querido, Acta endocr., K'hvn. 4; 79 (1950). - 2. Hoxt, R. E. und M. G. Levine, J. Clin. Endocr., Springfield 10, 101 (1950). - 3. LuGG, J. W. H. und J. M. Bowness, Austral. J. exp. Biol. med. Sci. 35, 395 (1957). - 4. Hamburger, C., Acta endocr., K'hvn. 17, 116 (1954). - 5. Devis, R., Bruxelles-méd. 29, 1568 (1949). - 6. Hollander, F., B. KRISS, E. KLEMpNer und R. T. Frank, Endocrinology 33, 217 (1943). - 7. McHeNRY, E. W., E. M. Semmons, R. Pearse und E. G. Meyer, Cancer Res. 7, 534 (1947). - 8. Laroche, G., A. Corteel und J. Delor, Ann. endocr., Paris 7, 191 (1946). - 9. Norymberski, J. K., R. D. Stubbs und H. F. West, Lancet, London I, 1276 (1953). 10. Callow, N. H., R. K. Callow und C. W. Emmens, Biochem. J. 32, 1312 (1938). - 11. Talbot, N. B., R. A. Berman und E. A. Mclachlan, J. biol. Chemistry 143, 211 (1942). - 12. Appleby, J. I., G. Gibson, J. K. Norymberski und R. D. Stubbs, Biochem. J. 60, 453 (1955). - 13. Popper, H., E. MANDel und H. Mayer, Biochem. Z. 291, 354 (1937). - 14. LindER, A., Statistische Methoden, 3. Aufl., Birkhäuser, Basel (1960). - 15. MEDGYESI, G. und K. Katona, Ärztl. Lab. 7, 247 (1961).

Professor Dr. R. Borth St. Michael's Hospital Toronto 2, Ontario Canada Professor Dr. H. Breuer 53 Bonn-Venusberg

\section{Photometrische Bestimmung der „klassischen“ Leucinaminopeptidase im Blutplasma und -serum}

\author{
Von R. J. HASChEN, W. FARR und D. Reichelt \\ Aus dem Institut für Klinische Biochemie der Martin-Lutber-Universität, Halle/Saale \\ (Direktor: Prof. Dr. med. R.J. Hascben)
}

(Eingegangen am 14. November 1966)

Es wird eine neue photometrische Methode zur Bestimmung der Leucinaminopeptidase („LAP“) im Serum beschrieben, die aufgrund ihrer Empfindlichkeit und Einfachheit für Routinezwecke geeignet ist. Als Substrat dient DL-Leucinhydrazid, das durch Serum-LAP bei pH 10,0 optimal zu Hydrazin und L-Leucin hydrolysiert wird. Das freigesetzte Hydrazin wird mit p-Dimethylaminobenzaldehyd in salzsaurer Lösung zu einem orangeroten Farbsalz umgesetzt, dessen Konzentration der enzymatischen Aktivität proportional ist und bei $455 \mathrm{~nm}$ photometriert wird. Die Bestimmung bei $\mathrm{pH} \mathrm{9,25} \mathrm{mit} \mathrm{optimaler} \mathrm{Mg}^{2+}$-Aktivierung ist wegen des größeren Zeitaufwandes für Routinezwecke weniger geeignet. - Die Normalwerte der LAP-Aktivität wurden im Serum und Plasma von Blutspendern bestimmt.

A new photometric method is described for the determination of leucine aminopeptidase ("LAP") in serum. It is sufficiently sensitive and simple for routine purposes. The substrate is DL-leucine hydrazide, which is hydrolysed by serum LAP optimally at $\mathrm{pH} 10.0$ to hydrazine and L-leucine. The released hydrazine is reacted with p-dimethylamino-benzaldehyde in hydrochloric acid solution, to give an orange-red salt. The concentration of the salt is proportional to the enzymic activity, and it is measured photometrically by its absorption at $455 \mathrm{~nm}$. At $\mathrm{pH} 9.25$ with optimal $\mathrm{Mg}^{2+}$-activation, longer time is required and the method is less suitable for routine measurements. The normal levels of LAP were determined in the serum and plasma from blood banks.

Die Leucinaminopeptidase „LAP“ (L-Leucylpeptidhydrolase, EC 3.4.1.1) des Serums scheint nach bisherigen Erfahrungen $(1,2,3)$ ein empfindlicher Indikator von Leberparenchymschäden zu sein. Von dieser „,klassischen " LAP (4) ist die Aminosäure-Arylamidase (5-9) zu unterscheiden, die in vielen Arbeiten zu Unrecht als
LAP bezeichnet wurde. Sie wurde vor allem bei intraund extrahepatischer Obstruktion erhöht gefunden (10). Als spezifisches Substrat der LAP gilt L-Leucinamid (4). Leucin- und vor allem Alanin-p-nitroanilid (11) und $-\beta$-naphthylamid (12) werden dagegen vorwiegend oder ausschließlich von der Aminosäure-Arylamidase hydro- 